EPJ Web of Conferences 85, 02002 (2015)

DOI: $10.1051 /$ epjconf/ 20158502002

(C) Owned by the authors, published by EDP Sciences, 2015

\title{
Limits of TMD evolution in SIDIS at moderate $Q$
}

\author{
Leonard Gamberg ${ }^{1, a}$ \\ ${ }^{1}$ Division of Science, Penn State University Berks, Reading, Pennsylvania 19610, USA
}

\begin{abstract}
We investigate semi-inclusive deep inelastic scattering measurements in the region of relatively small $Q$, where sensitivity to nonperturbative transverse momentum dependence can dominate the evolution. Using SIDIS data from the COMPASS experiment, we find that regions of coordinate space that dominate in TMD processes when the hard scale is of the order of only a few $\mathrm{GeV}$ are much larger than those commonly probed in large $Q$ measurements. This suggests that the details of nonperturbative effects in TMD evolution are especially significant in the region of intermediate $Q$. We emphasize the strongly universal nature of the nonperturbative component of evolution. and its potential to be tightly constrained by fits from a wide variety of observables that include both large and moderate $Q$.
\end{abstract}

\section{Introduction}

TMD factorization separates a transversely differential cross section into a perturbatively calculable part and several well-defined universal factors [1], including the transverse momentum parton distribution functions fragmentation functions. The latter are interpreted in terms of hadronic structure. For example, the TMD factorization theorems for semi-inclusive deep inelastic scattering (SIDIS) is schematically:

$$
\begin{aligned}
d \sigma_{\text {SIDIS }} & =\sum_{f} \mathcal{H}_{f, \text { SIDIS }}\left(\alpha_{s}(\mu), \mu / Q\right) \otimes F_{f / H_{1}}\left(x, k_{1 T} ; \mu, \zeta_{1}\right) \\
& \otimes D_{H_{2} / f}\left(z, k_{2 T} ; \mu, \zeta_{2}\right)+Y_{\text {SIDIS }}
\end{aligned}
$$

where similar expressions hold for Drell-Yan scattering (DY), and inclusive $e^{+} e^{-}$annihilation into back-to-back hadrons $\left(e^{+} e^{-} \rightarrow H_{1}+H_{2}+X\right)$. The first term is a generalized product of three factors which closely resemble a literal TMD parton model description. The factor, $\mathcal{H}\left(\alpha_{s}(\mu), \mu / Q\right)$ is the hard part, specific to the process, with the other two factors being the universal TMD PDFs and/or FFs, $F_{f / H_{1,2}}$ and $D_{H_{1,2} / f}$ (Note, in the case of "Todd" TMDs, like the Sivers function [2], universality is predicted to be generalized [3, 4]). The kinematical arguments, $Q, x_{1,2}$ and $z_{1,2}$ have standard definitions which can be found, for example, in Ref. [1]. The TMDs contain a mixture of both perturbative and nonperturbative contributions. But regardless of whether or not they are predominantly described by perturbative or nonperturbative behavior, they are universal, and so may be regarded as being associated with individual specific hadrons. The $Y_{\text {SIDIS }}$ term in Eq. (1) provides corrections for the region of large transverse momentum of order $Q$ where a description in terms of factorized TMD functions is no longer appropriate.

\footnotetext{
ae-mail: lpg10@psu.edu
}

In the QCD evolution of TMDs, the evolution kernel includes both a perturbative short-distance contribution as well as a large-distance nonperturbative, but strongly universal contribution. A unique aspect of TMD evolution is that the kernel for the evolution itself becomes nonperturbative in the region of large transverse distances. Moreover, one of the important predictions of the TMD factorization theorem [1], and a central component to the analysis of TMD evolution, is that the nonperturbative contribution to evolution is totally universal, not only with respect to different processes, but also with respect to the species of hadrons involved. Furthermore, the soft evolution is independent of whether the TMDs are PDFs or FFs, and is independent of whether the hadrons and/or partons are polarized. ${ }^{1}$ Therefore, a parametrization of the nonperturbative evolution from the $Q$ dependence in one observable strongly constrains the evolution of many other observables across a wide and diverse variety of different kinds of experiments and for both TMD PDFs and TMD FFs. This strong form of universality is, therefore, an important basic test of the TMD factorization theorem. It is related to the soft factors - the vacuum expectation values of Wilson loops - that are needed in the TMD definitions for consistent factorization with a minimal number of arbitrary cutoffs. Constraining the nonperturbative component of the evolution probes fundamental aspects of soft QCD.

Semi-inclusive deep inelastic scattering experiments are particularly suited to an examination of the variation in transverse momentum distributions with relatively low $Q$ and approximately fixed $x, z$ and $Q$ [5]. The COMPASS experiment has released data [6] for charged hadron SIDIS measurements that are differential in all kinematical parameters and cover a range of moderately low values of $Q$. We will use this to study the $Q$ dependence in the region

\footnotetext{
${ }^{1}$ The only dependence is on whether the target partons are quarks or gluons.
} 
of small $Q$ within the same experiment and for approximately fixed $x, z$. The species of hadrons in Ref. [6] is not fixed; the target is ${ }^{6} \mathrm{LiD}$ while the measured final state particles include all positively (negatively) charged hadrons. While the range in $Q$ in Ref. [6] is too small to allow for reasonably accurate fits to the nonperturbative evolution, it is significant enough that we can use it to rule out any dramatic variations with $Q$ that might be suggested by direct direct extrapolations from large $Q$ fits, and to infer certain general aspects of the very large coordinate space $Q$ dependence [7].

\section{TMD Evolution}

We summarize the basic formulas of TMD factorization [1]. The transversely differential cross section for SIDIS in transverse coordinate space, corresponding to Eq. (1), takes the form:

$$
\begin{gathered}
\frac{d \sigma}{d P_{T}^{2}} \propto \mathcal{H}_{f, \text { SIDIS }}\left(\alpha_{s}(\mu), \mu / Q\right) \int d^{2} \mathbf{b}_{T} e^{i \mathbf{b}_{T} \cdot \mathbf{P}_{T}} \tilde{F}_{H_{1}}\left(x, b_{T} ; \mu, \zeta_{1}\right) \\
\tilde{D}_{H_{2}}\left(z, b_{T} ; \mu, \zeta_{2}\right)+Y_{\text {SIDIS }}
\end{gathered}
$$

We work with the coordinate space integrand in the first term in Eq. (2) and also make the simplifying assumption of quark flavor independence, so we have dropped flavor indices; flavor dependence is easily restored in later formulae. ${ }^{2}$ The kinematical variables $x, z$ and $Q$ for SIDIS are defined in the usual way, and correspond to those of Ref. [6]. In our notation, $P$ is the four momentum of the produced hadron, $Q^{2}=-q^{2}$ where $q$ is the virtual photon momentum, $x=Q^{2} /\left(2 P_{H_{1}} \cdot q\right)$ where $P_{H_{1}}$ is the incoming hadron four-momentum, and $z=P_{H_{1}} \cdot P /\left(P_{H_{1}} \cdot q\right) . P_{T}$ is the transverse momentum of the produced hadron in a frame where both the incoming hadron and the virtual photon have zero transverse momentum.

The TMDs in Eqs. (2) contain dependence on the renormalization scale $\mu$ and $\zeta$, which are used to parametrize how the effects of soft gluon radiation are partitioned between the two TMDs [1]. In full QCD, the auxiliary parameters are exactly arbitrary and this is reflected in the the Collins-Soper (CS) equations for the TMD PDF,

$$
\frac{\partial \ln \tilde{F}\left(x, b_{T} ; \mu, \zeta_{1}\right)}{\partial \ln \sqrt{\zeta_{1}}}=\tilde{K}\left(b_{T} ; \mu\right),
$$

and the renormalization group ( $R G)$ equations

$$
\begin{gathered}
\frac{d \tilde{K}\left(b_{T} ; \mu\right)}{d \ln \mu}=-\gamma_{K}\left(\alpha_{s}(\mu)\right), \\
\frac{d \ln \tilde{F}\left(x, b_{T} ; \mu, \zeta_{1}\right)}{d \ln \mu}=\gamma_{\mathrm{PDF}}\left(\alpha_{s}(\mu) ; \zeta_{1} / \mu^{2}\right),
\end{gathered}
$$

and similarly for the FFs. The anomalous dimensions $\gamma_{K}\left(\alpha_{s}(\mu)\right)$ and $\gamma_{F}\left(\alpha_{s}(\mu) ; \zeta_{F} / \mu^{2}\right)$ are perturbatively calculable, and we will keep up to order $\alpha_{s}$ terms. The CS kernel, $\tilde{K}\left(b_{T} ; \mu\right)$, is also perturbatively calculable as long as $b_{T} \ll \sim 1 / \Lambda_{\mathrm{QCD}}$. Further, $\zeta_{1}$ and $\zeta_{2}$ which are not independent and are related to the physical hard scale $Q$

\footnotetext{
${ }^{2}$ See, however, Ref. [5].
}

via $\sqrt{\zeta_{1} \zeta_{2}}=Q^{2}$. In full QCD, the auxiliary parameters are exactly arbitrary, though to optimize the convergence properties of perturbatively calculable parts, a choice of $\mu \sim \sqrt{\zeta_{1}} \sim \sqrt{\zeta_{2}} \sim Q$ can be made. Over short transverse distance scales, $1 / b_{T}$ is the hard scale, and the transverse coordinate dependence in the TMD PDFs can itself be calculated in perturbation theory. With the choice of renormalization scale $\mu \sim 1 / b_{T}, \alpha_{s}\left(\sim 1 / b_{T}\right)$ approaches zero for small sizes due to asymptotic freedom ensuring that the small size transverse coordinate dependence is optimally calculable in perturbation theory. For very large $b_{T}$, the transverse coordinate dependence corresponds to intrinsic nonperturbative behavior associated with the hadron wave function where a prescription is needed to tame the growth of $\alpha_{s}\left(1 / b_{T}\right)$ and match to a nonperturbative, large distance description of the $b_{T}$-dependence. The renormalization group scale is therefore chosen to be $\mu_{b} \equiv C_{1} /\left|\mathbf{b}_{*}\left(b_{T}\right)\right|$, where $\mathbf{b}_{*}(b)$ is a function of $b_{T}$ that equals $b_{T}$ at small $b_{T}$, but freezes in the limit where $b_{T}$ becomes nonperturbatively large, i.e., when $b_{T}$ is larger than some fixed $b_{\max }$. This function must obey

$$
\mathbf{b}_{*}\left(\mathbf{b}_{T}\right)= \begin{cases}\mathbf{b}_{T} & b_{T} \ll b_{\max } \\ \mathbf{b}_{\max } & b_{T} \gg b_{\max }\end{cases}
$$

The most common taming prescription and the one that we will adopt here is $\mathbf{b}_{*}\left(\mathbf{b}_{\mathrm{T}}\right) \equiv \mathbf{b}_{\mathrm{T}} / \sqrt{1+b_{T}^{2} / b_{\max }^{2}}$. The factor $C_{1}$ is arbitrary and can be chosen to minimize higher order corrections. It is typically fixed at $C_{1}=2 e^{-\gamma_{\mathrm{E}}}$. To put Eq. (2) into a convenient form for perturbative calculations, we express each TMD function evolved from the reference scale $\mu_{b}$. Further since the purpose of this work is to investigate the large $b_{T}$ behavior at relatively small $Q$, we define,

$$
g_{\mathrm{PDF}}\left(x, b_{T} ; b_{\max }\right) \equiv g_{1}\left(x, b_{T} ; b_{\max }\right)-\ln \left(\tilde{F}_{H_{1}}\left(x, b_{*} ; \mu_{b}, \mu_{b}^{2}\right)\right)
$$

(and similarly for the FF) where $\tilde{F}_{H_{1}}\left(x, b_{T} ; \mu_{b}, \mu_{b}^{2}\right)$ has optimal perturbative behavior at small $b_{T}$. It is calculable, via an operator product expansion, in terms of collinear PDFs and Wilson coefficients with powers of small $\alpha_{s}\left(\mu_{b}\right)$ and perturbative coefficients that are well-behaved in the limit of $Q \gg \Lambda_{\mathrm{QCD}}$ (and contain no large logs of $b_{T}$ ). Then, we have for the TMD PDF

$$
\begin{gathered}
\tilde{F}_{H_{1}}\left(x, b_{T} ; Q, Q^{2}\right)=\exp \left\{-g_{\mathrm{PDF}}\left(x, b_{T} ; b_{\max }\right)\right. \\
-g_{K}\left(b_{T} ; b_{\max }\right) \ln \left(\frac{Q}{Q_{0}}\right)+\ln \left(\frac{Q}{\mu_{b}}\right) \tilde{K}\left(b_{*} ; \mu_{b}\right) \\
\left.+\int_{\mu_{b}}^{Q} \frac{d \mu^{\prime}}{\mu^{\prime}}\left[\gamma_{\mathrm{PDF}}\left(\alpha_{s}\left(\mu^{\prime}\right) ; 1\right)-\ln \left(\frac{Q}{\mu^{\prime}}\right) \gamma_{K}\left(\alpha_{s}\left(\mu^{\prime}\right)\right)\right]\right\},
\end{gathered}
$$


with a similar expression for the FF. Using these TMDs in Eq. (2) gives the cross section in the compact form:

$$
\begin{aligned}
\frac{d \sigma}{d P_{T}^{2}} & \propto \text { F.T. } \exp \left\{-g_{\mathrm{PDF}}\left(x, b_{T} ; b_{\mathrm{max}}\right)-g_{\mathrm{FF}}\left(z, b_{T} ; b_{\mathrm{max}}\right)\right. \\
& -2 g_{K}\left(b_{T} ; b_{\max }\right) \ln \left(\frac{Q}{Q_{0}}\right)+2 \ln \left(\frac{Q}{\mu_{b}}\right) \tilde{K}\left(b_{*} ; \mu_{b}\right) \\
& +\int_{\mu_{b}}^{Q} \frac{d \mu^{\prime}}{\mu^{\prime}}\left[\gamma_{\mathrm{PDF}}\left(\alpha_{s}\left(\mu^{\prime}\right) ; 1\right)+\gamma_{\mathrm{FF}}\left(\alpha_{s}\left(\mu^{\prime}\right) ; 1\right)\right. \\
& \left.\left.-2 \ln \left(\frac{Q}{\mu^{\prime}}\right) \gamma_{K}\left(\alpha_{s}\left(\mu^{\prime}\right)\right)\right]\right\}+Y_{\mathrm{SIDIS}} .
\end{aligned}
$$

The functions $g_{\mathrm{PDF}}\left(x, b_{T} ; b_{\max }\right)$ and $g_{\mathrm{FF}}\left(z, b_{T} ; b_{\max }\right)$ parametrize the intrinsic large $b_{T}$ behavior associated with the TMD PDF and the TMD fragmentation function respectively. They are independent of $Q$. In our notation, they also include, via the definitions Eqs. (7), and the analogous express for the FF [7], the matching to the small $b_{T}$ behavior that is calculable using collinear factorization. The function $g_{K}\left(b_{T} ; b_{\max }\right)$ on the second line is the correction to the CS kernel at large $b_{T}$ which includes nonperturbative effects. The underlying simplicity in TMD evolution in that there is a single universal function $\tilde{K}\left(b_{T} ; \mu\right)$ that governs the evolution of the cross section at small $P_{T}$, though in Eq. (9) it has been split into three parts: $\gamma_{K}\left(\alpha_{s}\left(\mu^{\prime}\right)\right), \tilde{K}\left(b_{*} ; \mu_{b}\right)$, and $g_{K}\left(b_{T} ; b_{\max }\right)^{3}$.

With the $b_{T}$-dependence of the perturbatively calculable part of Eq. (9) frozen above a certain $b_{\max }$, the remaining evolution is described by the function $g_{K}\left(b_{T} ; b_{\max }\right)$, which is totally universal and independent of $Q, x$, or $z$. $g_{K}\left(b_{T} ; b_{\max }\right)$ generally contains both perturbatively calculable contributions and nonperturbative effects. By its definition [1], it must vanish like a power at small $b_{T}$. Detailed studies of power corrections in Refs. [8-11] suggest that it vanish like $b_{T}^{2}$ (or an even power of $b_{T}$ ) as $b_{T} \rightarrow 0$.

The value of $b_{\max }$, as well as the functional form for the matching $\mathbf{b}_{*}\left(\mathbf{b}_{\mathrm{T}}\right)$, is exactly arbitrary in full QCD. Practically speaking however, dependence on $b_{\max }$ typically does arise due to incomplete knowledge of the exact form of $g_{K}\left(b_{T} ; b_{\max }\right)$ at large $b_{T}$. It is preferable to choose it to be large enough to maximize the perturbative content of the calculation, while small enough that only a solidly perturbative range of $b_{T}$ is included in the calculation of $\tilde{K}\left(b_{*} ; \mu_{b}\right)$. A frequently used ansatz for $g_{K}\left(b_{T} ; b_{\max }\right)$ is

$$
g_{K}\left(b_{T} ; b_{\max }\right)=g_{2}\left(b_{\max }\right) \frac{1}{2} b_{T}^{2},
$$

where $g_{2}\left(b_{\max }\right)$ is a Gaussian fit parameter. This choice for $g_{K}\left(b_{T} ; b_{\max }\right)$, if positive and reasonably large, imposes a very strong Gaussian suppression of the nonperturbative regions of $b_{T}$ in Eq. (9) whenever $Q$ becomes significantly larger than $Q_{0}$. As implied by the notation, $g_{2}\left(b_{\max }\right)$ should be expected to take on different values depending on the choice of $b_{\max }$.

${ }^{3}$ The TMD term in Eq. (9) is derived using the approximation that $P_{T} \ll Q$. For an accurate calculation of the full cross section, a correction term, the $Y$-term, is need for the region $P_{T} \sim Q$; symbolized by the last term in Eq. (9). We neglect it, focusing only on the TMD term: a common practice in studies done at moderate $Q$.
In Ref. [12], parametrizations of the TMD PDFs were constructed out of previous nonperturbative fits within the updated version of the Collins-Soper-Sterman (CSS) formalism of Ref. [1], and were presented in a form where the contributions to separate operator definitions of the TMD PDFs and fragmentation functions could be automatically identified. These parametrizations were constructed from nonperturbative functions that were extracted in earlier work in the old version of the CSS formalism for DY scattering $[13,14]$, and were combined with fixed scale SIDIS fits at low $Q$ that arose in the context of hadronic structure studies [15]. A direct extrapolation of the DY fits to low $Q$ gives evolution that is too rapid [16], and in Ref. [12] this was conjectured to be due to the role of larger $x$ in the small $Q$ fits, so an $x$-dependent function was inserted to obtain a fit that interpolated between all of the fits, within the TMD evolution formalism.

Recently it was illustrated [16] that the rapid evolution given by extrapolating the nonperturbative extractions from DY cross sections at large $Q$ is too fast to account very generally for data in the region of $Q$ of order a few $\mathrm{GeV}$. Thus we have revisited the details of the nonperturbative contribution to evolution in the region of small $Q$ [7]. To maintain consistency with the general aim of extracting properties intrinsic to specific hadrons we would ideally vary $Q$ while holding $x, z$, and hadron species fixed. In experiments, however, these variables are correlated, and practical fitting becomes challenging. In the next section, we use the multi-differential COMPASS data from Ref. [6] to study the variation in the multiplicity distribution with small variations in $Q$ and roughly fixed $x$ and $z$ bins within the same experiment.

\section{Empirical Evolution at Moderate $Q$}

Empirically, the SIDIS data in Ref. [6] reveal that the differential cross section as a function of $P_{T}$ is reasonably well-described by a Gaussian functional form in the region of small $P_{T}$ (see, e.g., Fig. 4 of Ref. [6]), with a width that broadens very slightly with increasing $Q$. We have quantified this rate of change within the language of TMD evolution [7]. We then use Eq. (9) to estimate how well it matches the change in widths of the Gaussian fits under different assumptions for $g_{K}\left(b_{T} ; b_{\max }\right)$.

In Ref. [6], the data for hadron multiplicities are fitted using a Gaussian form $d \sigma / d P_{T}^{2} \propto \exp \left\{-P_{T}^{2} /\left\langle P_{T}^{2}\right\rangle\right\}$ and the resulting $\left\langle P_{T}^{2}\right\rangle$ values are presented. Expressed in terms of the two dimensional Fourier transform (F.T.) in $b_{T}$-space,

$$
\frac{d \sigma}{d P_{T}^{2}} \propto \text { F.T. } \exp \left\{-\frac{b_{T}^{2}\left\langle P_{T}^{2}\right\rangle}{4}\right\} .
$$

Therefore to match to the evolved formula, Eq. (9), we assume that all the terms in the exponent of Eq. (9) can be approximated as quadratic; that is $g_{\mathrm{PDF}}\left(x, b_{T} ; b_{\max }\right)$ and $g_{\mathrm{FF}}\left(z, b_{T} ; b_{\max }\right) \propto b_{T}^{2}{ }^{4}$. For the moderate $Q$ range

\footnotetext{
${ }^{4} \mathrm{~A}$ note of caution is needed here because the actual behavior of $g_{\mathrm{PDF}}\left(x, b_{T} ; b_{\max }\right)$ and $g_{\mathrm{FF}}\left(z, b_{T} ; b_{\max }\right)$ includes, via the definitions in Eq. (7), non-power law effects from collinear perturbation theory that
} 
of the COMPASS data we consider, where a Gaussian fit actually provides a good description of the data, we work with the conjecture that the small $b_{T}$ behavior from $g_{\mathrm{PDF}}\left(x, b_{T} ; b_{\mathrm{max}}\right)$ and $g_{\mathrm{FF}}\left(z, b_{T} ; b_{\mathrm{max}}\right)$ is negligible. However, the details of the initial-scale treatment of $g_{\mathrm{PDF}}\left(x, b_{T} ; b_{\mathrm{max}}\right)$ and $g_{\mathrm{FF}}\left(z, b_{T} ; b_{\mathrm{max}}\right)$ may become important when extending to much larger $Q$.

A result of CS evolution is that, for the TMD term, the $Q$-dependence of the logarithm of the $b_{T}$-dependence is linear in $\ln (Q)$ [17]. We therefore define,

$\tilde{\sigma}_{\mathrm{TMD} \text { term }} \equiv \mathcal{H}\left(\alpha_{s}(Q)\right) \tilde{F}_{H_{1}}\left(x, b_{T} ; Q, Q^{2}\right) \tilde{D}_{H_{2}}\left(z, b_{T} ; Q, Q^{2}\right)$.

which is the Fourier transform of the TMD term in Eq. (2). Then, CSS evolution of the TMDs gives

$$
\left.\frac{d \ln \tilde{\sigma}_{\mathrm{TMD} \text { term }}}{d \ln Q^{2}}\right|_{\mathrm{b}_{\mathrm{T}} \text { dep }}=\left.\tilde{K}\left(b_{T} ; \mu_{0}\right)\right|_{\mathrm{b}_{\mathrm{T}} \text { dep }},
$$

where the right side is independent of $Q, x$ and $z$. Assuming that the $Y$-term can be neglected, and using Eq. (11), we then make the approximation that

$$
\tilde{\sigma}_{\mathrm{TMD} \text { term }} \approx \exp \left\{-\frac{b_{T}^{2}\left\langle P_{T}^{2}\right\rangle}{4}\right\} .
$$

For small $P_{T}$, the $P_{T}$-shape of the data in Ref. [6] is empirically observed to broaden slightly as $Q$ increases, but remains quite well described by a Gaussian parametrization. Therefore, maintaining the Gaussian shape as $Q$ varies, Eq. (11) must take the form

$$
\frac{d \sigma}{d P_{T}^{2}} \propto \text { F.T. } \exp \left\{-\frac{b_{T}^{2}}{4}\left(\left\langle P_{T}^{2}\right\rangle_{0}+4 C_{\text {evol }} \ln \left(\frac{Q_{2}}{Q_{1}}\right)\right)\right\} .
$$

Here, $\left\langle P_{T}^{2}\right\rangle_{0}$ may depend only on $x$ and $z$ (it is independent of $Q)$ and $C_{\text {evol }}$ is a numerical parameter that is, in principle, independent of $x$ and $z . Q_{1}$ and $Q_{2}$ are initial and final hard scales. If $x$ and $z$ are held fixed, then the variation of $\left\langle P_{T}^{2}\right\rangle$ with $Q$ can be found directly from the $b_{T}$-space integrand in Eq. (15):

$$
\Delta\left\langle P_{T}^{2}\right\rangle\left(Q_{1}, Q_{2}\right) \approx 4 C_{\mathrm{evol}} \ln \left(\frac{Q_{2}}{Q_{1}}\right),
$$

where we define $\Delta\left\langle P_{T}^{2}\right\rangle\left(Q_{1}, Q_{2}\right)=\left\langle P_{T}^{2}\right\rangle_{Q=Q_{2}}-\left\langle P_{T}^{2}\right\rangle_{Q=Q_{1}}$. We use Eq. (16) to extract approximate bounds on $C_{\text {evol }}$ from experimental results for $\Delta\left\langle P_{T}^{2}\right\rangle\left(Q_{1}, Q_{2}\right)$.

The only aspect of TMD factorization that we have used so far is Eq. (13). Specifically, we have applied it to the case of the COMPASS data for the small range of $Q$ where the $P_{T}$ distribution appears to remain approximately Gaussian even after evolution to obtain Eq. (15). At this stage, we do not address the question of whether $\tilde{K}\left(b_{T} ; \mu_{0}\right)$ is governed primarily by perturbative or nonperturbative $b_{T}$-dependence. Further, while $C_{\text {evol }}$ resembles $g_{2}$ in a quadratic approximation to $g_{K}\left(b_{T} ; b_{\max }\right)$, here it should be emphasized that it is meant merely to approximate the collective effect of all the $Q$-dependent terms in

are important for accurately describing the small $b_{T}$ region. This corresponds to the behavior of the large $P_{T}$ tail, and accounting for it properly would involve a careful treatment of the $Y$ term as well.

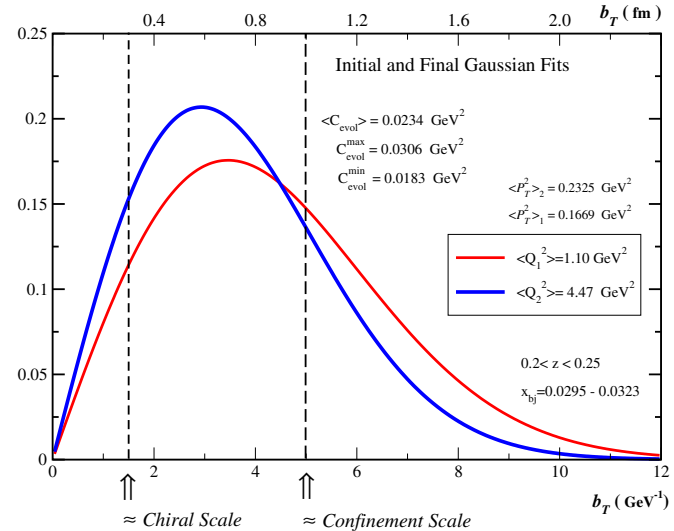

Figure 1. Coordinate space Gaussian fits showing the largest variation in the width found in Tables I and II [7] with a change from $\sqrt{\left\langle Q_{1}^{2}\right\rangle}=1.049 \mathrm{GeV}$ to $\sqrt{\left\langle Q_{2}^{2}\right\rangle}=2.114 \mathrm{GeV}$. The precise function being plotted is Eq. (17) with the initial (red) and final (thick blue) $\left\langle P_{T}^{2}\right\rangle$ COMPASS values in Eq. (18). The peak moves toward smaller values with increasing $Q$. We mark the approximate chiral symmetry breaking scale from Ref. [18] at $b_{T} \approx 1.5 \mathrm{GeV}^{-1}$ and the approximate confinement scale at $b_{T} \approx 5.0 \mathrm{GeV}^{-1}$

the exponent of Eq. (9), in a way consistent with Eq. (13), and it should not be identified at this stage with any specific perturbative or nonperturbative terms. Of course, perturbative contributions are not quadratic, so the quadratic ansatz for the right side of Eq. (13) is a poor one for small $b_{T}$. We nevertheless attempt to use it to capture the general $Q$-dependence of the $P_{T}$-width in the vicinity of small $Q$ variations where the data appear from [6] to be reasonably well-described by Gaussian fits. We will further analyze the reliability of such an approximation. Since the right side of Eq. (13) is universal and $x, Q$, and $z$ independent, then a test of the universal value for $C_{\text {evol }}$ probes the assumptions that led to the use of Eq. (15) as a model, such as the Gaussian functional form and the neglect of the $Y$ term.

In a full treatment of evolution, there is also a $Q$ dependence that affects only the normalization of the cross section. Since we are mainly interested in the variation in the width, we ignore any such contributions and focus only on the broadening of the Gaussian shape.

\section{$4 P_{T}$-Broadening \& Relevance of Large $b_{T}$}

Evolution leads to a well-known broadening of the $P_{T}$ width with $Q$ at fixed $x$ and $z$. For a significant effect to be clearly observable, one must examine fixed $x$ and $z$ bins over sufficiently broad ranges of $Q$. In Ref. [6], Figs. 5 and 6 allow $Q$ intervals of order $\sim 1.0 \mathrm{GeV}$ for fixed $x$ and $z$ bins to be identified across several bins in $Q$. In each panel, the fifth and sixth columns of vertical blocks correspond to fixed $x$ and $z$ bins with four and five $Q^{2}$-bins, respectively. Since these give the maximum variation in $Q$, they are the data we will use to obtain conservative limits on the amount of evolution at moderately small $Q$. In Ref. [7] we show the results for $C_{\text {evol }}$ from $\Delta\left\langle P_{T}^{2}\right\rangle\left(Q_{1}, Q_{2}\right)$ for each $x$ and $z$ bin. 


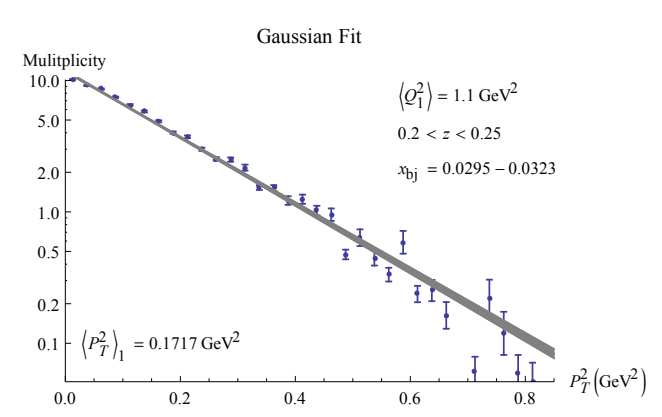

(a)



(c)

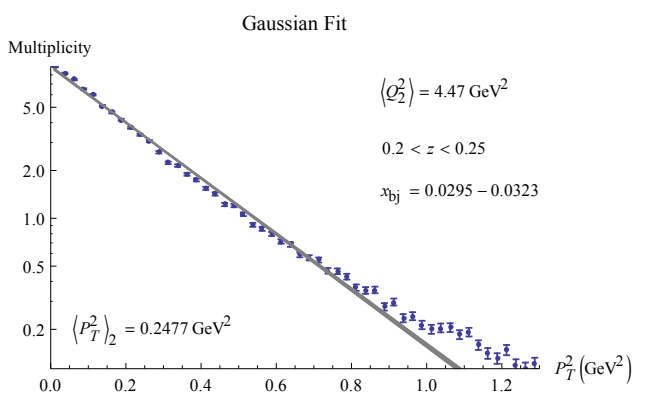

(b)

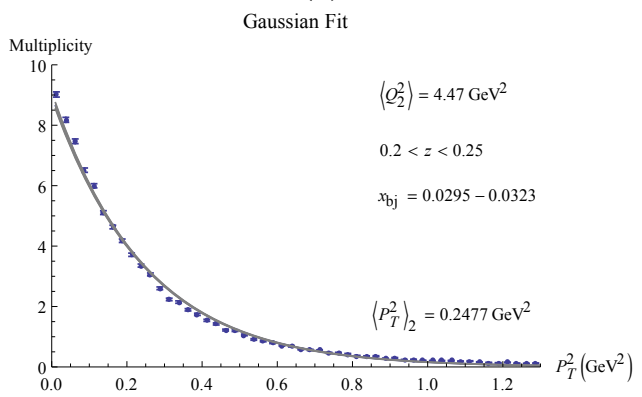

(d)

Figure 2. (a) Gaussian fit for $Q=1.049 \mathrm{GeV}$, all $P_{T}$. (b) Gaussian fit for $Q=2.114 \mathrm{GeV}$, all $P_{T}$. (c, d) Same as (a, b) but on a linear axis. The gray band represents a $99 \%$ confidence band for the fit parameters, where only the reported statistical errors have been included.

A limitation of our analysis is the unavoidably large $Q$ bin sizes relative to $Q$ itself in the moderate $Q$ region. We estimated the error from large $Q$ bin sizes on $C_{\text {evol }}$ using three methods. We direct the reader to Ref. [7] for details. The trend in Tables I and II and Fig. 2, suggests a small yet non-vanishing $Q$-dependence in the $P_{T}$ width; the lowest value of $C_{\mathrm{evol}}$ is $0.0040 \mathrm{GeV}^{2}$ and the largest value is $0.0306 \mathrm{GeV}^{2}$.

The question of the relevance of the nonperturbative region in the Collins TMD-factorization theorem may be addressed directly in the context of the COMPASS measurements by using the fits to estimate the important range of $b_{T}$. We have plotted the fits obtained by the COMPASS collaboration [6] in coordinate space as the solid lines in Fig. 1. Since the transverse momentum space distribution is obtained from a two dimensional Fourier transform from the coordinate space expression, we have also included a factor of $b_{T}$. Also, since we are primarily interested in the width of the distribution, we normalize to unity in the integration over $b_{T}$. Instead of Eq. (14) the curves in Fig. 1 are for

$$
\frac{b_{T}\left\langle P_{T}^{2}\right\rangle}{2} \exp \left\{-\frac{b_{T}^{2}\left\langle P_{T}^{2}\right\rangle}{4}\right\} .
$$

Thus, up to a normalization, Eq. (17) is the integrand of the Fourier transform to coordinate space for the region of small $P_{T}$.

The initial and final Gaussian slope parameters $\left\langle P_{T}^{2}\right\rangle$ that we have used in Fig. 1 correspond to the largest parameter $C_{\text {evol }}$ that is found in Tables I and II [7], yielding an estimate of the maximum reasonable rate of variation in the width with changes in $Q$ of order $\sim 1.0 \mathrm{GeV}$ and so is consistent with a strategy of placing rough upper limits on the rate of evolution that can reasonably be expected at low $Q$. The largest value for $C_{\text {evol }}$ corresponds to the second row of the first entry in Table II, and the corresponding slope parameters from Ref. [6] are:

$$
\begin{aligned}
& \left\langle P_{T}^{2}\right\rangle_{Q_{1}=1.049 \mathrm{GeV}}=0.1669 \pm 0.0012 \mathrm{GeV}^{2} ; \\
& \left\langle P_{T}^{2}\right\rangle_{Q_{2}=2.114 \mathrm{GeV}}=0.2325 \pm 0.0011 \mathrm{GeV}^{2},
\end{aligned}
$$

where the uncertainties are the quoted statistical uncertainties from the fit only.

The resulting curves shown in Fig. 1 are peaked around $b_{T} \sim 3.0 \mathrm{GeV}^{-1}$ with tails extending out to nearly $b_{T} \sim$ $10.0 \mathrm{GeV}^{-1}$, i.e. up to transverse sizes about twice that of the proton charge radius, suggesting that the effect of nonperturbative input is substantial, at least in this region of moderate $Q$. From the general features of Fig. 1, we conclude that, for the differential cross section in the limit of $P_{T} \rightarrow 0$, the relevant range of $b_{T}$ is likely to be nearly dominated by the nonperturbative region of $b_{T}$ for $Q \sim$ $1.0 \mathrm{GeV}$ to $\sim 2.0 \mathrm{GeV}$.

The robustness of this conclusion might be questioned on the grounds that the fits from [6] apply to a restricted range, $P_{T}<0.85 \mathrm{GeV}$. One could speculate that including more of the large $P_{T}$ tail might result in an enhanced relative contribution from small $b_{T}$. To address this, we have performed our own fit of the Gaussian form using the same data from Ref. [6] that gave the two curves for $Q=1.049 \mathrm{GeV}$ and $Q=2.114 \mathrm{GeV}$ in Fig. 1, but now for the entire range of $P_{T}$ (up to $P_{T} \gtrsim 1.0 \mathrm{GeV}$ ). We perform the fitting in Wolfram Mathematica. The new Gaussian fits are shown in Fig. 2. From the plot, it is clear that the values we find for the Gaussian slopes, $\left\langle P_{T}^{2}\right\rangle_{Q_{1}=1.049 \mathrm{GeV}}$ and $\left\langle P_{T}^{2}\right\rangle_{Q_{2}=2.114 \mathrm{GeV}}$, are so close to the COMPASS values that the curves in Fig. 1 are nearly unchanged, despite the in- 
clusion of larger $P_{T}$. Instead of Eq. (18), we find:

$$
\begin{aligned}
& \left\langle P_{T}^{2}\right\rangle_{Q_{1}=1.049 \mathrm{GeV}}^{\text {New Fits }}=0.1717 \pm 0.0011 \mathrm{GeV}^{2} ; \\
& \left\langle P_{T}^{2}\right\rangle_{Q_{2}=2.114 \mathrm{GeV}}^{\text {New Fits }}=0.2477 \pm 0.0008 \mathrm{GeV}^{2},
\end{aligned}
$$

where again the uncertainties are statistical uncertainties from the fit only. The difference between the COMPASS fits in Eq. (18) and our fits in Eq. (19) for $Q_{1}=$ $1.049 \mathrm{GeV}$ is $0.0048 \mathrm{GeV}^{2}$ and for $Q_{2}=2.114 \mathrm{GeV}$ it is $0.0152 \mathrm{GeV}^{2}$. This difference gives a sense of the systematic uncertainty due to the upper cutoff on $P_{T}$

To see how the new fits affect the coordinate space distribution, Eq. (17), we have replotted in Fig. 3 the original curves from Fig. 1 along with the curves using the new parameters in Eq. (19). It is clear that neglecting the large $P_{T}$ values has little influence on the general features of the fits discussed above; namely, that there is a large contribution from intervals of $b_{T}$ deep in the nonperturbative region.

A critique could be made regarding the use of a Gaussian form on the grounds that analyticity considerations [19] imply a power law fall-off for the large $P_{T}$ behavior of TMD correlation functions. Moreover, a power law behavior $1 / P_{T}^{2}$ (up to logarithmic corrections and the effects of evolution of collinear PDFs) is a prediction of pQCD (see, for example, Ref. [5]). This power law behavior is tied to singular behavior in the transverse position at small $b_{T} \cdot{ }^{5}$ Figure 2(b) shows that the Gaussian form does have some slight difficulty accounting for the full range of $P_{T}$ for the larger $Q_{2}=2.114 \mathrm{GeV}$ value. To address this, we have refitted the $Q_{2}=2.114 \mathrm{GeV}$ data using a Kaplan functional form:

$$
\frac{d \sigma}{d P_{T}^{2}} \propto\left(1+\frac{P_{T}^{2}}{M_{\mathrm{kap}}^{2}}\right)^{-v} .
$$

The result, shown in Fig. 4, gives a slightly more successful fit than the Gaussian fit of Fig. 2(b). When switching from the Gaussian fit to the Kaplan fit it is possible to quantify the goodness of the two fits. We use a straightforward coefficient of determination, $R^{2}$, which is defined in the usual way [20] as $1-\mathrm{SS}_{\mathrm{res}} / \mathrm{SS}_{\mathrm{T}}$, where $\mathrm{SS}_{\mathrm{res}}$ is the residual sum of squares of each data point and the fit and $\mathrm{SS}_{\mathrm{T}}$ is the total sum of squares. This coefficient is a simple measure of the goodness of the fit that approaches unity for a perfect fit. In this case, the $R^{2}$ fit parameter rises modestly from 0.9918 to 0.9988 when moving from the Gaussian form to the Kaplan fit. The final Kaplan fit parameters are $M_{\text {kap }}^{2}=1.3006 \mathrm{GeV}^{2}$ and $v=6.7216$. For the lower value of $Q=1.049 \mathrm{GeV}$, the Gaussian form actually gives a better fit than the Kaplan form. Indeed, from Fig. 2(a) it can be seen that even the Gaussian fit tends to overshoot the data slightly at large $P_{T}$. This could be due to the role of resonances at very small $Q$.

As with the Gaussian form, we may examine the Kaplan fit in coordinate space. Instead of Eq. (17) we have

$$
\frac{2 b_{T}^{v} M_{\text {kap }}}{\Gamma(v)}\left(\frac{M_{\text {kap }}}{2}\right)^{v} K_{1-v}\left(b_{T} M_{\text {kap }}\right),
$$

${ }^{5}$ The true large $P_{T}$ behavior of the TMD functions is not directly meaningful at very large $P_{T}$, since TMD factorization (without the $Y$ term) is inapplicable once the $P_{T}$ is comparable with $Q$. Clearly, the $Y$ term will be need be incorporated in the future to deal with these issues. where $K_{1-v}$ is the order $1-v$ modified Bessel function of the second kind. In coordinate space, the difference

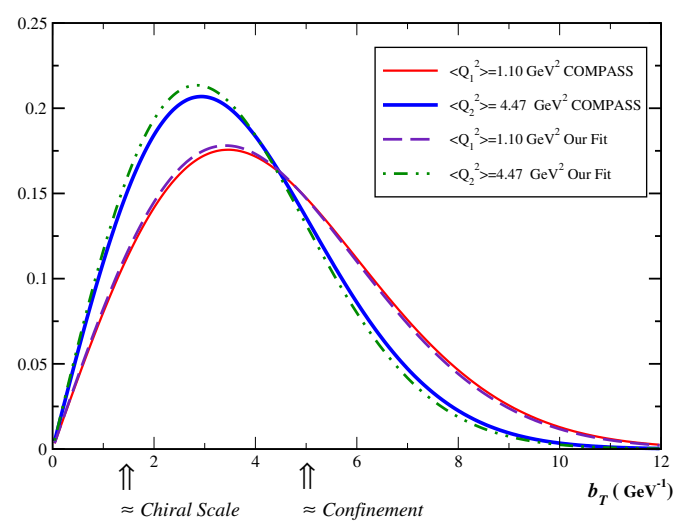

Figure 3. Gaussian fits again showing the largest variation in the width found in Tables I and II [7]. The solid red and thick blue curves are the same as those in Fig. 1, in which the fit is restricted to the region of $P_{T} \leq 0.85 \mathrm{GeV}$. The purple dashed and green dot-dashed curves are from the refit Gaussian curves in Fig. 2 that use all $P_{T}$ and correspond to Eq. (17)with the initial and final $\left\langle P_{T}^{2}\right\rangle$ from Eq. (19).

between the Gaussian and the Kaplan fits can be examined by comparing Eq. (21) and Eq. (17) with the fit parameters corresponding to $Q_{2}=2.114 \mathrm{GeV}$. It can be seen that an analysis of the important regions of $b_{T}$ leads to roughly the same conclusions as in the case of the Gaussian fit (see Figs. $5 \mathrm{a}-\mathrm{d}$ ). We conclude that regions of $b_{T}$ deep into the nonperturbative regime are significant - is robust for $P_{T} \rightarrow 0$ and for $Q \sim 1 \mathrm{GeV}$ to $\sim 2 \mathrm{GeV}$, regardless of which functional form is used.

\section{Comparison with TMD Evolution}

Next, we examine the evolved formula in Eq. (9) to estimate how well it matches the change in widths of the Gaussian fits under different assumptions for $g_{K}\left(b_{T} ; b_{\max }\right)$. Consider the coordinate space factor in Eq. (9) of the TMD term, including a factor of $b_{T}$ in analogy with (17):

$$
\begin{aligned}
& \frac{b_{T}}{N(Q)} \exp \{-g_{\mathrm{PDF}}\left(x, b_{T} ; b_{\max }\right)-g_{\mathrm{FF}}\left(z, b_{T} ; b_{\max }\right) \\
&-2 g_{K}\left(b_{T} ; b_{\max }\right) \ln \left(\frac{Q}{Q_{0}}\right)+2 \ln \left(\frac{Q}{\mu_{b}}\right) \tilde{K}\left(b_{*} ; \mu_{b}\right) \\
&+\int_{\mu_{b}}^{Q} \frac{d \mu^{\prime}}{\mu^{\prime}}\left[\gamma_{\mathrm{PDF}}\left(\alpha_{s}\left(\mu^{\prime}\right) ; 1\right)+\gamma_{\mathrm{FF}}\left(\alpha_{s}\left(\mu^{\prime}\right) ; 1\right)\right. \\
&\left.\left.-2 \ln \left(\frac{Q}{\mu^{\prime}}\right) \gamma_{K}\left(\alpha_{s}\left(\mu^{\prime}\right)\right)\right]\right\},
\end{aligned}
$$

where $N(Q)$ is a normalization factor so that the full quantity integrates to unity. We will require that for $Q=Q_{0}=$ $1.049 \mathrm{GeV}$, Eq. (22) reduces to the $Q=1.049 \mathrm{GeV}$ COMPASS Gaussian fit shown in Fig. 1. which fixes the input functions, $-g_{\mathrm{PDF}}\left(x, b_{T} ; b_{\max }\right)-g_{\mathrm{FF}}\left(z, b_{T} ; b_{\max }\right)$, such that Eq. (22) reduces exactly to Eq. (17) at $Q=Q_{0}$ [7].

We use the one-loop $\overline{\mathrm{MS}}$ expressions for the anomalous dimensions with $C_{1}=2 e^{-\gamma_{E}}$, which are included in 


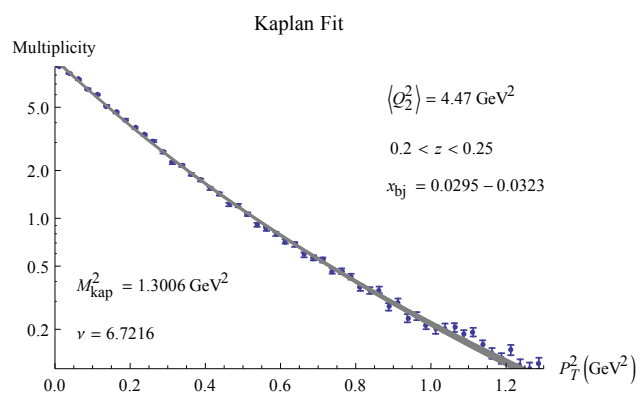

(a)

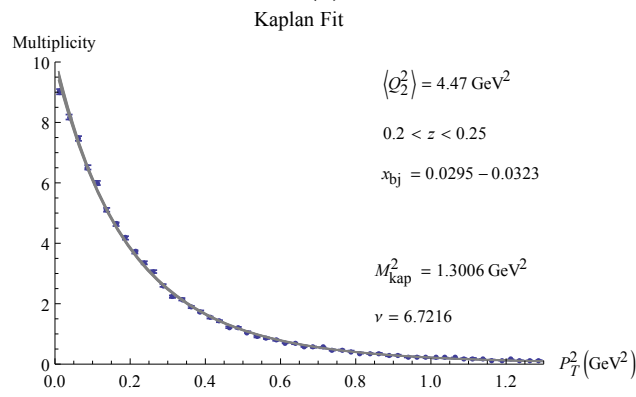

(b)

Figure 4. Fits of the Kaplan function, Eq. (20), for $Q=2.114 \mathrm{GeV}$ and for all $P_{T}$ with (a) a logarithmic plot and (b) a linear plot. The gray band represents a $99 \%$ confidence band for the fit parameters, where only the reported statistical errors have been included. (Color online.)

Appendix A of Ref. [7] for reference. We use the approximation $\alpha_{s}(\mu)=1 / 2 \beta_{0} \ln \left(\mu / \Lambda_{\mathrm{QCD}}\right)$ for the running coupling with 3 flavors and $\Lambda_{\mathrm{QCD}}=0.2123 \mathrm{GeV}$ Then, the integrals in the one loop anomalous dimensions may be straightforwardly evaluated to obtain analytic expressions for all perturbative parts of the exponent in Eq. (22) explicit expression is given in App. C of Ref. [7].

For $g_{K}\left(b_{T} ; b_{\max }\right)$, we start by using Eq. (10), with a conservative $b_{\max }=0.5 \mathrm{GeV}^{-1}$ and several sample values of $g_{2}\left(b_{\max }\right)$. We compare with the maximum observed rate of evolution seen in the COMPASS data - the curves already shown in Fig. 1. The results are shown in Fig. 5(a) and (c), where the dot-dashed curves show the evolution to $Q^{2}=4.47 \mathrm{GeV}^{2}$ for a range of sample values for $g_{2}$.

We begin with $g_{2}=0$ and see essentially no effect on the $b_{T}$ distribution when $Q$ is varied; the integrand is small in the region of small $b_{T}$ where perturbative evolution would be substantial, and setting $g_{2}=0$ suppresses any nonperturbative contribution to evolution. Next, we consider $g_{2}=C_{\text {evol }}$, with the maximum value of $C_{\text {evol }}=0.0306 \mathrm{GeV}^{2}$. Finally, we consider $g_{2}=0.1 \mathrm{GeV}^{2}$ and $g_{2}=0.7 \mathrm{GeV}^{2}$ which are values more typical of fits obtained at large $Q$, as well as the renormalon analysis value of $g_{2}=0.19 \mathrm{GeV}^{2}$ in Ref. [9]. (See, also, Fig. 1 of Ref. [14].)

We repeat this exercise for the much more liberal value of $b_{\max }=2.0 \mathrm{GeV}^{-1}$, and the result is shown in Figs. 5 (b) and (d). In Figs. 5(a)-(d), a value of $g_{2}\left(b_{\max }\right) \lesssim C_{\text {evol }}^{\max }$ is clearly preferred over values of $g_{2}\left(b_{\max }\right) \geq 0.1 \mathrm{GeV}^{2}$. Note that with $g_{2}=0$, there is very weak evolution in the $b_{T}$ shape relative to the variations in the width suggested by the COMPASS data in the small range of $Q$ values. A choice of $g_{2}=C_{\text {evol }}^{\max }=0.0306 \mathrm{GeV}^{2}$ is roughly consistent with the upper limit on the rate of evolution observed in Tables I and II of Ref. [7]. Thus, if we demand the ansatz in Eq. (10) for the form of $g_{K}\left(b_{T} ; b_{\max }\right)$ for all $b_{T}$, then we estimate that the true value of $g_{2}$, must lie roughly in the range of $0<g_{2} \lesssim 0.03 \mathrm{GeV}^{2}$.

\section{Modified Large $b_{T}$ Behavior}

Because of the strong universality of $g_{K}\left(b_{T} ; b_{\max }\right)$, the results of the last section seem on the surface to indicate a discrepancy between the low $Q$ data and successful fits of the past that focus on larger $Q$, which tend to find $g_{2} \gtrsim$ $0.1 \mathrm{GeV}^{2}[13,14,21,22]$. For instance, values of $g_{2}$ have been found to be as large as $0.68 \mathrm{GeV}^{2}$ [13], and a value of $g_{2}=0.19 \mathrm{GeV}^{2}$ is used in Ref. [22] for SIDIS in the CSS formalism, both using a value of $b_{\max }=0.5 \mathrm{GeV}^{-1}$. However, the quadratic ansatz in Eq. (10) seems to impose excessive suppression of the very large nonperturbative $b_{T}$ region whenever $g_{2} \gtrsim 0.1 \mathrm{GeV}^{2}$.

To resolve the apparent discrepancy discussed above, we recall that large $Q$ fits, e.g. for $Q \gtrsim 10 \mathrm{GeV}$, are sensitive mainly to the region of $b_{T} \lesssim 2.0 \mathrm{GeV}^{-1}$. See, for example, Fig. 4 of Ref. [14] and compare this with Fig. 1, where contributions from $b_{T} \gtrsim 2.0 \mathrm{GeV}^{-1}$ dominate. Now let us assume that nonperturbative effects become totally dominant at some large size scale $b_{\mathrm{NP}}$, where $g_{K}\left(b_{T} ; b_{\max }\right)$ acquires a more complicated and as-yet unknown precise form. Recall also that $g_{K}\left(b_{T} ; b_{\max }\right)$ is predicted to vanish as a power of $b_{T}^{2}$ at small $b_{T}$ [8-11]. Thus, for $b_{T} \ll b_{\mathrm{NP}}$ the following expansion applies:

$$
g_{K}\left(b_{T} ; b_{\max }\right)=a_{1}\left(\frac{b_{T}^{2}}{b_{\mathrm{NP}}^{2}}\right)+a_{2}\left(\frac{b_{T}^{4}}{b_{\mathrm{NP}}^{4}}\right)+\cdots .
$$

We conjecture that large $Q$ fits typically obtain a large $g_{2}$ because they are sensitive only to the first power-law correction in Eq. (23). By contrast, at smaller $Q$ higher powers, and eventually the complete functional form, become important.

We propose that the optimal way to proceed is to use a functional form for $g_{K}\left(b_{T} ; b_{\max }\right)$ that: a) respects its strong universality set forth in TMD factorization by matching to earlier large $Q$ fits that use a Gaussian form but b) avoids strong disagreement with the results of the empirical analysis of SIDIS data from Sect. 4. Thus, we impose the following conditions: (i) At small $b_{T}^{2}$, the lowest order coefficient in Eq. (23), i.e. $a_{1} / b_{\mathrm{NP}}^{2}$, must be roughly $\gtrsim 0.1 \mathrm{GeV}^{2}$ in order to be consistent with the values of $g_{2} / 2$ found in Ref. $[9,13,14,21,22]$, thereby respecting the strong universality of $g_{K}\left(b_{T} ; b_{\max }\right)$. (ii) At $b_{T} \gg b_{\mathrm{NP}}, g_{K}\left(b_{T} ; b_{\max }\right)$ should become nearly constant, or at most logarithmic in $b_{T}$. As a simple example, we propose [7]

$$
g_{K}\left(b_{T} ; b_{\max }\right)=\frac{g_{2}\left(b_{\max }\right) b_{\mathrm{NP}}^{2}}{2} \ln \left(1+\frac{b_{T}^{2}}{b_{\mathrm{NP}}^{2}}\right) .
$$

Expanding around $b_{T} \ll b_{\mathrm{NP}}$ gives the first two terms,

$$
g_{2}\left(b_{\max }\right) \frac{1}{2} b_{T}^{2}-g_{2}\left(b_{\max }\right) \frac{1}{4 b_{\mathrm{NP}}^{2}} b_{T}^{4}+\cdots .
$$




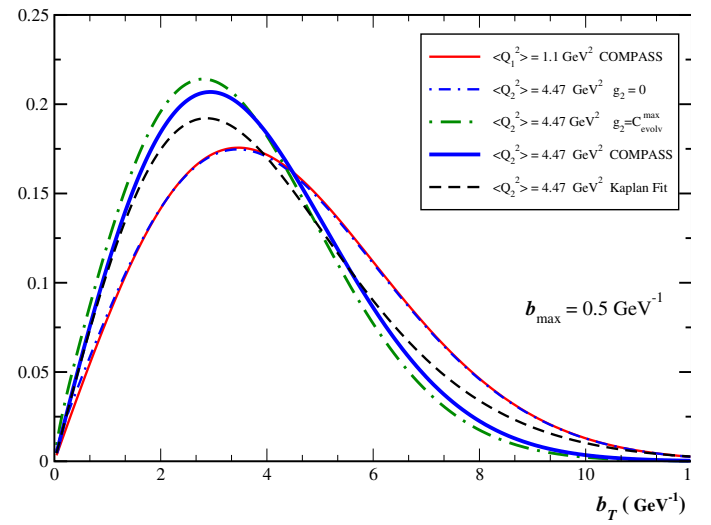

(a)

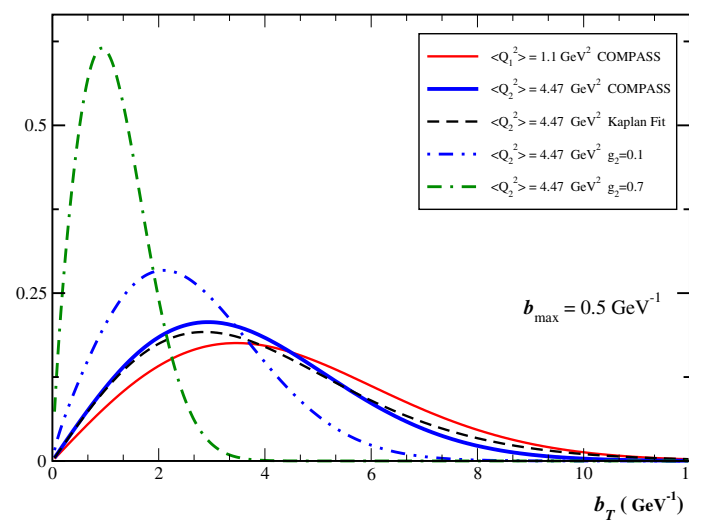

(c)

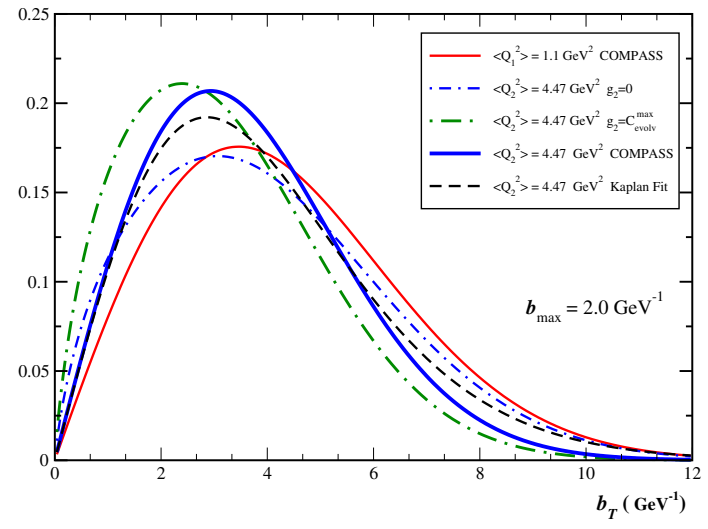

(b)

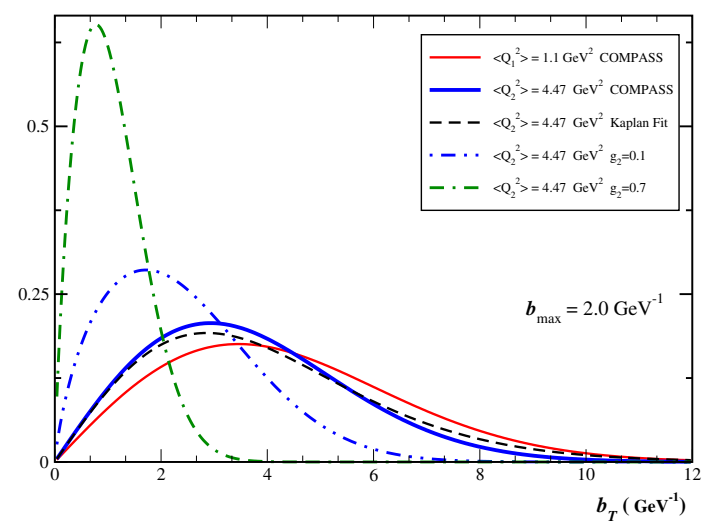

(d)

Figure 5. Left Panels (a) and (c): The solid red and thick blue lines are the same initial and final Gaussian fits obtained by COMPASS as in Fig. 1 for $Q_{1}^{2}=1.1$ GeV ${ }^{2}$ and $Q_{2}^{2}=4.47 \mathrm{GeV}^{2}$ respectively. The black dashed curve is the Kaplan fit for $Q^{2}=4.47 \mathrm{GeV}^{2}$, shown in [7]. The dot-dashed lines are the TMD factorization expression in Eq. (22) for the evolution to $Q_{2}^{2}=4.47 \mathrm{GeV}^{2}$ with the Gaussian ansatz from Eq. (10) for $g_{K}\left(b_{T} ; b_{\max }\right)$ with $b_{\max }=0.5 \mathrm{GeV}^{-1}$. The positions of the peaks of the evolved distributions decrease with increasing $g_{2}$ : Figure (a) shows the results for $g_{2}=0$ (blue dot-dashed) and $C_{\mathrm{evol}}^{\max }=0.0306 \mathrm{GeV}^{2}$ (green dot-dashed); Figure (c) shows the result for $g_{2}=0.1 \mathrm{GeV}^{2}$ (blue dot-dashed) and $g_{2}=0.7 \mathrm{GeV}^{2}$ (green dot-dashed). All curves are normalized to one in the integration over $b_{T}$. Right Panels (b) and (d): Same as the left panels, but for $b_{\max }=2.0 \mathrm{GeV}^{-1}$

In Fig. 6 we illustrate how the low $Q$ dependence of the COMPASS data may be accommodated into earlier larger $Q$ fits by using the modified $g_{K}\left(b_{T} ; b_{\max }\right)$ from Eq. (24) with $b_{\max }=0.5 \mathrm{GeV}^{-1}, g_{2}=0.1 \mathrm{GeV}^{2}$ and $b_{\mathrm{NP}}=$ $2.0 \mathrm{GeV}^{-1}$. Since the lowest order term in the expansion in Eq. (25) matches Eq. (10) with $g_{2}=O\left(0.1 \mathrm{GeV}^{2}\right)$ and thus is generally consistent with earlier fits such as Ref. [21, 22]. In this way, moderate $Q$ data may be accommodated without introducing disagreement with important and universal nonperturbative contributions obtained in earlier fits, while simultaneously giving access to further universal nonperturbative information. For now we propose Eq. (24) only as a simple example of how $g_{K}\left(b_{T} ; b_{\max }\right)$ might possibly be modified at very large $b_{T}$. In practice, better and more detailed parametrizations may be needed, possibly obtainable from nonperturbative studies (see e.g. [23]).
By allowing a more general treatment of the nonperturbative component of the CS kernel than the usual power law, we find that we may extend TMD factorization to lower $Q$ SIDIS measurements with no need to distort the perturbative part of evolution that is necessary to unify low $Q$ cross section measurements with large $Q$ ones. Moreover, we are able to maintain consistency with prior successful fits of nonperturbative parts done at larger $Q$. In addition to the recalling the important role of the $Y$-term, a possible limitation of TMD studies such as this one, done at such small $Q$, is that they may begin to approach the boundary of the region of applicability for the TMD factorization formalism. However, the general trends that we observe are dramatic enough that we expect our main conclusions to be robust. Even considering the issues related to the $Y$-term and possible limitations of TMD-factorization at very low $Q$ discussed above, it is difficult to reconcile the small values of $C_{\text {evol }}$ found in Tables I and II [7] 


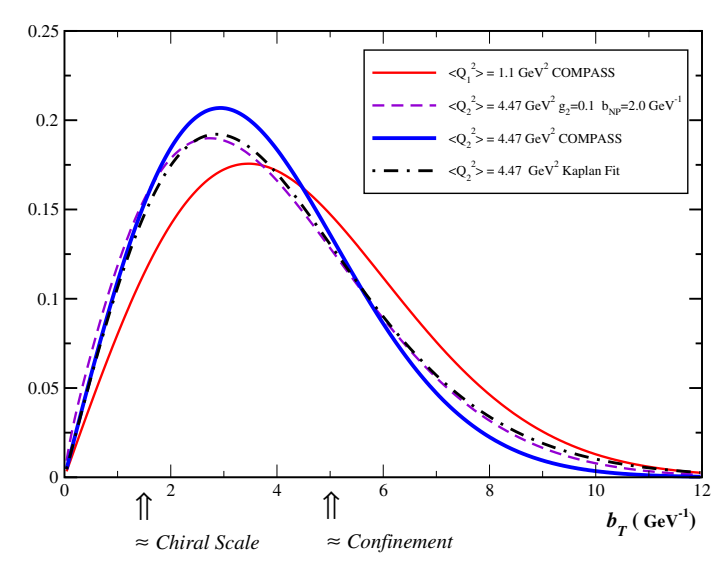

Figure 6. The solid red and thick blue curves are again the same initial and final Gaussian fits obtained by COMPASS for $Q^{2}=1.1 \mathrm{GeV}^{2}$ and $Q^{2}=4.47 \mathrm{GeV}^{2}$ respectively - the same as in Fig. 1 . The black dot-dashed curve is the Kaplan fit for $Q^{2}=4.47 \mathrm{GeV}^{2}$. For comparison, the purple short-dashed curve is the TMD factorization expression in Eq. (22), but now using Eq. (24) for $g_{K}\left(b_{T} ; b_{\max }=\right.$ $0.5 \mathrm{GeV}^{-1}$ ) with $b_{\mathrm{NP}}=2.0 \mathrm{GeV}^{-1}$ and $g_{2}=0.1 \mathrm{GeV}^{2}$. This should be compared with the $g_{2} \geq 0.1 \mathrm{GeV}^{2}$ curves in Fig. 5 where the quadratic ansatz for $g_{K}\left(b_{T} ; b_{\max }\right)$, Eq. (10) is used.

with the much larger nonperturbative soft evolution found from direct extrapolations of global fits of Drell-Yan or large $Q$ processes to much lower $Q$, if one limits the treatment of the nonperturbative large $b_{T}$ evolution factor to the quadratic form in Eq. (10) for all $b_{T}$. In this regard, we confirm one of the main observations of Ref. [16]. The meaning that we extract from these observations however is very different. In our analysis, we find much greater sensitivity to the details of the nonperturbative large $b_{T}$ structure, rather than evidence that nonperturbative contributions to evolution are unnecessary. By contrast, it has been suggested in Refs. [16, 24], within the context of similar but alternative evolution formalisms, that accounting for nonperturbative evolution can be avoided entirely even at scales of order $Q \sim 1.0$ to $2.0 \mathrm{GeV}$. Moreover, we find that the complications that arise from extrapolating from large to moderate $Q$ arise because of the greater care necessary in treating the nonperturbative contribution to evolution as larger $b_{T}$ values become relevant to evolution, not because such non-perturbative effects are less relevant (see also and [25]). By accounting for the nonperturbative behavior at very large $b_{T}$, as discussed here, we find that it is not difficult to reconcile past large $Q$ fits of nonperturbative evolution with the moderate $Q$ fits as is displayed in Fig. 6.

\section{Acknowledgements}

I thank my collaborators, Christine Aidala, Bryan Field, and Ted Rogers for their work on this project. Discussions with Miguel Echevarria, Ahmed Idilbi, Ignazio Scimemi, and Andrea Signori are gratefully acknowledged. This work is supported by the U.S. Department of Energy, under contract No. DE-FG0207ER41460.

\section{References}

[1] J.C. Collins, Foundations of Perturbative $Q C D$ (Cambridge University Press, Cambridge, 2011)

[2] D.W. Sivers, Phys. Rev. D41, 83 (1990)

[3] J.C. Collins, Phys. Lett. B536, 43 (2002), hep-ph/0204004

[4] S.J. Brodsky, D.S. Hwang, I. Schmidt, Nucl. Phys. B642, 344 (2002), hep-ph/0206259

[5] A. Bacchetta, D. Boer, M. Diehl, P.J. Mulders, JHEP 08, 023 (2008), 0803.0227

[6] C. Adolph et al. (COMPASS), Eur.Phys.J. C73, 2531 (2013), 1305.7317

[7] C. Aidala, B. Field, L. Gamberg, T. Rogers, Phys.Rev. D89, 094002 (2014), 1401.2654

[8] G.P. Korchemsky, G.F. Sterman, Nucl.Phys. B437, 415 (1995), hep-ph/9411211

[9] S. Tafat, JHEP 0105, 004 (2001), hep-ph/0102237

[10] E. Laenen, G.F. Sterman, W. Vogelsang, Phys.Rev. D63, 114018 (2001), hep-ph/0010080

[11] E. Laenen, G.F. Sterman, W. Vogelsang, pp. 14111413 (2000), hep-ph/0010183

[12] S.M. Aybat, T.C. Rogers, Phys. Rev. D83, 114042 (2011), 1101.5057

[13] F. Landry, R. Brock, P.M. Nadolsky, C.P. Yuan, Phys. Rev. D67, 073016 (2003), hep-ph/0212159

[14] A.V. Konychev, P.M. Nadolsky, Phys.Lett. B633, 710 (2006), hep-ph/0506225

[15] P. Schweitzer, T. Teckentrup, A. Metz, Phys.Rev. D81, 094019 (2010), 1003. 2190

[16] P. Sun, F. Yuan, Phys.Rev. D88, 114012 (2013), 1308.5003

[17] J.C. Collins, D.E. Soper, G.F. Sterman, Nucl.Phys. B250, 199 (1985)

[18] P. Schweitzer, M. Strikman, C. Weiss, Acta Phys.Polon.Supp. 6, 109 (2013), 1212.4031

[19] P. Schweitzer, M. Strikman, C. Weiss, JHEP 1301, 163 (2013), 1210.1267

[20] R.E. Walpole, R.H. Myers, S.L. Myers, K.E. Ye, Probability and Statistics for Engineers and Scientists (9th Edition), 9th (Pearson, January 6, 2011)

[21] P.M. Nadolsky, D. Stump, C. Yuan, Phys.Rev. D61, 014003 (2000), hep-ph/9906280

[22] P.M. Nadolsky, D. Stump, C. Yuan, Phys.Rev. D64, 114011 (2001), hep-ph/0012261

[23] J. Collins (2014), 1409. 5408

[24] M.G. Echevarria, A. Idilbi, A. Schafer, I. Scimemi, Eur.Phys.J. C73, 2636 (2013), 1208. 1281

[25] U. D’ Alesio, M.G. Echevarria, S. Melis, I. Scimemi (2014), 1407.3311 